\title{
A gene toolbox for monitoring autophagy transcription
}

\author{
Matteo Bordi $\mathbb{D}^{1}$, Rossella De Cegli $\mathbb{D}^{2}$, Beatrice Testa ${ }^{3}$, Ralph A. Nixon ${ }^{4,5,6}$, Andrea Ballabio $\mathbb{( D}^{2,7,8}$ and Francesco Cecconi $\mathbb{D}^{1,3,9}{ }^{\bowtie}$
}

(c) The Author(s) 2021

Autophagy is a highly dynamic and multi-step process, regulated by many functional protein units. Here, we have built up a comprehensive and up-to-date annotated gene list for the autophagy pathway, by combining previously published gene lists and the most recent publications in the field. We identified 604 genes and created main categories: MTOR and upstream pathways, autophagy core, autophagy transcription factors, mitophagy, docking and fusion, lysosome and lysosome-related genes. We then classified such genes in sub-groups, based on their functions or on their sub-cellular localization. Moreover, we have curated two shorter sub-lists to predict the extent of autophagy activation and/or lysosomal biogenesis; we next validated the "induction list" by Real-time PCR in cell lines during fasting or MTOR inhibition, identifying ATG14, ATG7, NBR1, ULK1, ULK2, and WDR45, as minimal transcriptional targets. We also demonstrated that our list of autophagy genes can be particularly useful during an effective RNAsequencing analysis. Thus, we propose our lists as a useful toolbox for performing an informative and functionally-prognostic gene scan of autophagy steps.

Cell Death and Disease (2021)12:1044; https://doi.org/10.1038/s41419-021-04121-9

\section{INTRODUCTION}

Macroautophagy is a pathway of organelle or protein degradation via a typical vesicle, the autophagosome, that promotes recycling of essential cellular components [1]. This process is described as a flux, through which multiple complexes (often coded by autophagy-related genes, ATGs) tightly regulate each one of several sequential steps [2, 3], from an autophagosome nucleation to its fusion with lysosomes and the completion of substrate degradation. Macroautophagy (referred to hereafter as autophagy) can also function selectively, when it promotes degradation of a precise substrate by specific autophagy receptors, such as NDP52, NBR1 and p62/SQSTM1 [2] and by involving the fine coupling of numerous kinases (i.e., AMPK, MTOR, PINK1 and TBK1 among others) $[2,4,5]$. Hence, autophagosomes can also engulf, among other intracellular targets, mitochondria (mitophagy) [6], peroxisomes (pexophagy), ER (ER-phagy), lipid droplets (lipophagy), bacteria (xenophagy) [2], and centriolar satellites (doryphagy) [7]. Moreover, many recent studies discovered novel factors directly or indirectly involved in the regulation of autophagy, with their number constantly growing [8]. Of note, since autophagy activity has been linked to development and progression of a plethora of pathologies [9-11], it becomes increasingly fundamental to develop new strategies for the prediction of a specific autophagy status and/or step [8]. In the last few years, whole transcriptome analysis via RNA-seq technology followed by biological pathway enrichment analysis $[12]$ has been widely used to this end $[13,14]$. However, this type of analysis should rely on complete gene lists that accurately described the pathway of interest; to this aim, herein we have curated a comprehensive and up-to-date annotated gene list for autophagy pathway, by combining previously published gene lists [15], most recent publications in the field (reported in the excel file) [8] and bioinformatics tools. Indeed, a handful of autophagyrelated resources, available on-line, have been already created collecting diverse data type including functional, structural and biological information: Autophagy, Necrosis, ApopTosis OrchestratorS (THANATOS, http://thanatos.biocuckoo.org) [16], Human Autophagy Database (HADb, http://www.autophagy.lu/), and Human Autophagy Modulator Database (HAMdb, http://hamdb. scbdd.com) [17]. Here, we propose a novel updated gene list that dissects the autophagy pathway and includes the mitophagy process, which can be easily validated experimentally via in silico approaches. Our main objective was to generate an innovative toolbox for an informative and functionally-prognostic gene analysis of all steps of autophagy, by using RNA-seq, that could provide new insights into the molecular mechanisms that drive autophagy regulation or dysfunction. Moreover, starting from the main list of autophagy-related genes, we have curated, as a proof of concept, two shorter sub-lists to predict the two key stages of autophagy activation and lysosomal biogenesis. A so called "activation list" has been derived from the comparison of publications, in which we reported conditions of gene upregulation upon autophagy activation [18-27]. Also, we validated such activation list by Real-Time (qPCR). The "Iysosomal biogenesis

\footnotetext{
${ }^{1}$ Department of Pediatric Hemato-Oncology and Cell and Gene therapy, IRCCS Bambino Gesù Children's Hospital, Rome, Italy. ${ }^{2}$ Telethon Institute of Genetics and Medicine (TIGEM), Pozzuśoli, Naples, Italy. ${ }^{3}$ Department of Biology, University of Rome Tor Vergata, Rome, Italy. ${ }^{4}$ Center for Dementia Research, Nathan Kline Institute for Psychiatric Research, Orangeburg, NY, USA. ${ }^{5}$ Departments of Psychiatry, New York University School of Medicine, New York, NY, USA. ${ }^{6}$ Cell Biology, New York University School of Medicine, New York, NY, USA. ${ }^{7}$ Department of Molecular and Human Genetics and Neurological Research Institute, Baylor College of Medicine, Houston, TX, USA. ${ }^{8}$ Medical Genetics Unit,

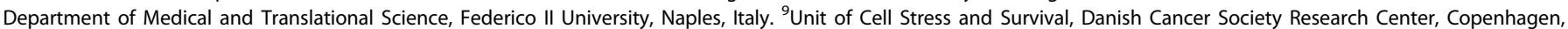
Denmark. ${ }^{凶}$ email: cecconi@cancer.dk

Edited by Professor Gerry Melino
}

Received: 10 May 2021 Revised: 15 July 2021 Accepted: 20 July 2021

Published online: 02 November 2021 
list" resulted instead from an extensive literature analysis, obtained by combining data that underwent previous experimental validation $[15,18,19,28-30]$. Further, we used the "autophagy core" list for gene set enrichment analysis by means of the published RNA-sequencing (RNA-seq) dataset, performed on starved cells [31] or on Down syndrome primary fibroblasts, in which it has been reported an mTORC1 hyperactivation associated to inhibition of autophagy induction [27], and thus demonstrating its effective applicability. Indeed, these two-tier experiments let us conclude that different approaches could be used to generate sub-lists of interests, related to the many steps of autophagy regulation and progression, according to each research need.

\section{RESULTS}

The 604 genes of our list belong to 6 main categories: MTOR and upstream pathways (135 genes), autophagy core (197 genes), autophagy regulators (68 genes), mitophagy (80 genes), docking and fusion (22 genes), lysosome (162 genes) and lysosome-related genes (34 genes) (Fig. 1, Supplementary Table 1 and Supplementary Figure 1). Within each category, we classified genes in groups based on their functions along the pathway (i.e., ATG8 Ubiquitinlike conjugation systems) or, in other cases, based on their subcellular localization (i.e., lysosome) or, lastly, based on the composition of specific complexes (such as MTOR complex 1 or ULK1 complex) (Fig. 1). The lysosome list is based on compartments database (https://compartments.jensenlab.org); moreover, we generated a customized lysosome-related gene list including genes essential for lysosomal activities whose lysosomal location has not yet been experimentally verified. Indeed, some genes belong to more than one category due to their multiple role (indicated in red in the Supplementary Table 1): for example, the regulator of vesicular transport "RAB7A" is located in i) the "HOPS" $[32]$, ii) the "Rab proteins involved in autophagosome formation" [33], and iii) the "mitophagy core" [34] sub-groups. Similarly, the pro-autophagy scaffold factor AMBRA1 is placed in both "Beclin1/ $\mathrm{PI3K}$ complex interacting proteins" and in "mitophagy core" subgroups. Furthermore, beyond the core ATG proteins critical for autophagosome formation, we classified some genes based on their effect on autophagy, thus creating lists of "Positive regulators of autophagy" and "negative regulators of autophagy" (refer to Supplementary Table 1). Also, we did the same for mitophagy. In addition, we listed mitochondria fission and fusion proteins, as well as specific transcription factors within the mitophagy category; in fact, mitochondrial dynamics and mitophagy are tightly inter-connected and it has been suggested that they regulate each-other $[6,35]$. Nonetheless, recent studies demonstrated that mitophagy induction is sustained by the concomitant activation of transcription factors, such as Transcription factor EB (TFEB) and TFE3 [36], that promote lysosomal and autophagic gene expressions. Hence, we performed on the entire list the Gene Ontology Enrichment Analysis (GOEA), by using the DAVID online, to obtain an over-representative analysis of the genes, divided into Biological Process terms, Cellular Compartment terms and Molecular Function terms (Supplementary Figure 2 and Supplementary Table 4).

Beside the list of 604 genes, we developed as a proof of concept, and by two different approaches two shorter gene lists to predict the activation status of autophagy and the key step of lysosomal biogenesis. In a first case, starting from experimental data available in the literature, we identified 20 targets related to autophagy activation and validated them by qPCR: ATG10, ATG14, ATG16L1, ATG3, ATG4, ATG7, ATG9A, BCL2, GABARAP, GABARAPL1, MAP1LC3B, NBR1, OPTN, PINK1, SQSTM1, ULK1, ULK2, UVRAG, WDR45, WIPI1 (Fig. 2) [18-27]. To this end we used two different cell lines (HEK-293 and SH-SY5Y). We induced autophagy in these systems by (i) classical starvation of amino acids and serum by incubation in EBSS [Earle's balanced salt solution (EBSS)] for $4 \mathrm{~h}$ (HEK-293) and $8 \mathrm{~h}$ (SH-SY5Y), or by treating them with AZD8055 (100 nM), a potent mTOR inhibitor [37], for $8 \mathrm{~h}$. Markedly,

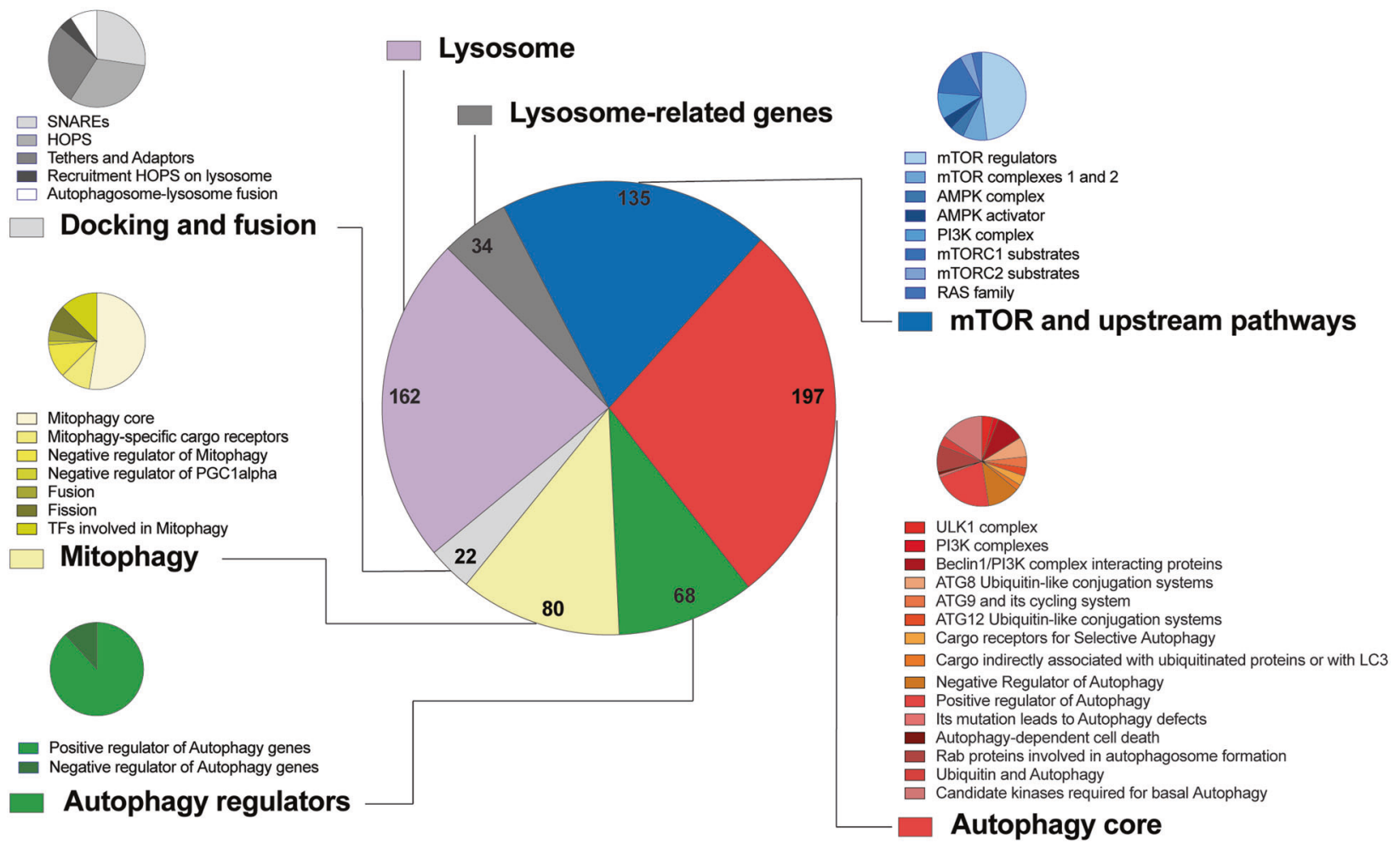

Fig. 1 Graphic representation of the $\mathbf{6 0 4}$ genes that compose the entire autophagic list. Genes were classified in 6 main groups: $\mathrm{mTOR}$ and upstream pathways (135 genes), autophagy core (197 genes), autophagy regulators (68 genes), mitophagy (80 genes), docking and fusion (22 genes), lysosome (162 genes) and lysosome-related genes (34 genes). For each group, also subgroups are reported. 


\begin{tabular}{|c|c|}
\hline Induction list & Lysosomal biogenesis \\
\hline ATG10 & ATP6V0B \\
\hline ATG14 & ATP6V0D1 \\
\hline ATG16L1 & ATP6V0D2 \\
\hline ATG3 & ATP6V0E1 \\
\hline ATG4 & ATP6V1C1 \\
\hline ATG7 & ATP6V1H \\
\hline ATG9A & CD63 \\
\hline BCL2 & CLCN3 \\
\hline GABARAP & CLCN7 \\
\hline GABARAPL1 & CTSA \\
\hline MAP1LC3B & CTSB \\
\hline NBR1 & CTSD \\
\hline OPTN & CTSF \\
\hline PINK1 & CTSS \\
\hline SQSTM1 & GAA \\
\hline ULK1 & GBA \\
\hline ULK2 & GLA \\
\hline UVRAG & GNS \\
\hline WDR45 & HEXA \\
\hline \multirow[t]{7}{*}{ WIPI1 } & LAMP1 \\
\hline & MCOLN1 \\
\hline & NEU1 \\
\hline & PSAP \\
\hline & SCPEP1 \\
\hline & TMEM55B \\
\hline & TPP1 \\
\hline
\end{tabular}

Fig. 2 The "induction list" and the "lysosomal biogenesis list". The two shorter lists were generated by identifying the gene targets related to autophagy activation (Induction list) and lysosomal biogenesis.

$4 \mathrm{~h}$ or $8 \mathrm{~h}$ EBSS treatments are interval times commonly used to induce autophagy; furthermore, we selected $8 \mathrm{~h}$ for AZD, since we have previously demonstrated that at this time point a significant elevation in the expression of several autophagy genes [27] can be detected. In order to map a universal "autophagy activation" signature, we next compared the responses of the two cell lines and identified those targets that could have been up-regulated in both conditions: we found that 15 genes out of the 20 were significantly up-regulated upon one or both conditions (Fig. 3A-C). qPCR analysis revealed that mRNA levels of ATG3, ATG9A, GABARAP, MAP1LC3B, SQSTM1, UVRAG and WIPI1 were significantly elevated only upon EBSS; while ATG10 and OPTN were transcriptionally induced only in the presence of AZD. Notably, the expression of ATG14, ATG7, NBR1, ULK1, ULK2 and WDR45 was highly increased by both treatments and in both cell lines (Fig. 3C), indicating that these genes may represent ideal targets for the evaluation of autophagy initiation by transcriptional analyses. Second, it is now clear that an efficient autophagic process requires the concomitant increase in lysosomal activity, for ensuring a proper degradation of autophagic substrates. Hence, for the second sub-lists, we identified 26 targets to assess the state of lysosomal biogenesis (Fig. 2) at variance with the autophagy activation list, this sub-list has been realized by only comparing data from recent work, in which mRNA levels of lysosomal genes in response to autophagy enhancement were accurately and
A

HEK 293

B
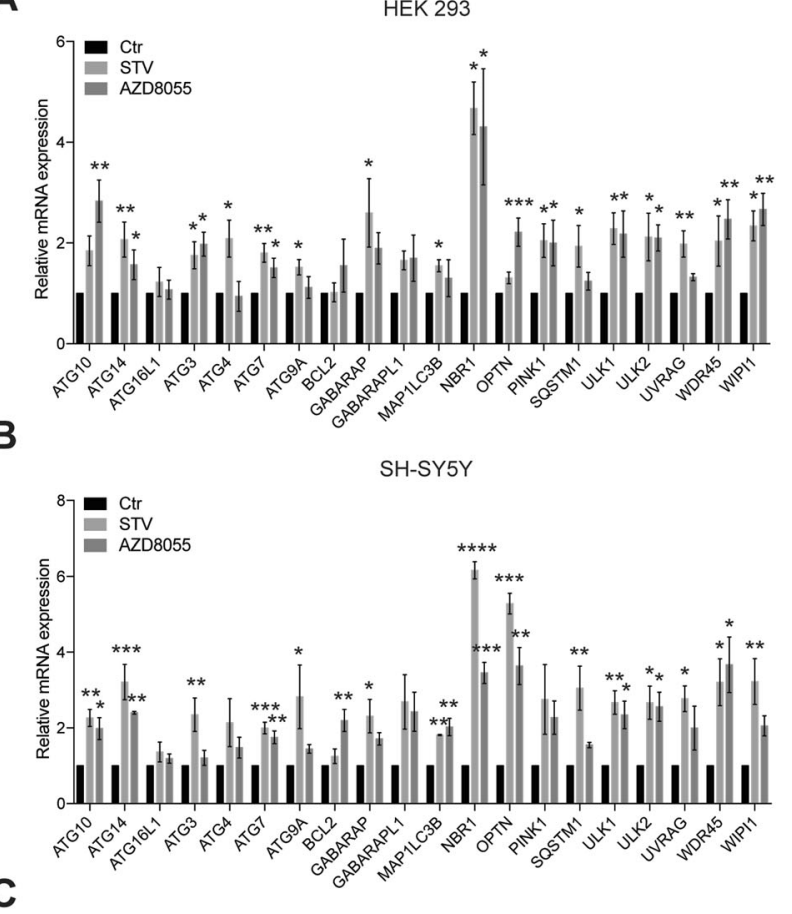

\begin{tabular}{|l|l|}
\hline Only upon STV & ATG3, ATG9A, GABARAP, MAP1LC3B, SQSTM1, UVRAG, WIPI1 \\
\hline Only upon AZD & ATG10, OPTN \\
\hline Upon both & ATG14, ATG7, NBR1, ULK1, ULK2, WDR45 \\
\hline
\end{tabular}

Fig. 3 Validation of the "induction list" by Real-time PCR in cell lines during fasting or MTOR inhibition. HEK-293 (A) and SH-SY5Y (B) cells were cultured in EBSS starvation medium for $4 \mathrm{~h}$ or treated with AZD8055 (100 nM) for $8 \mathrm{~h}$. ATG10, ATG14, ATG16L1, ATG3, ATG4, ATG7, ATG9A, BCL2, GABARAP, GABARAPL1, MAP1LC3B, NBR1, OPTN, PINK1, SQSTM1, ULK1, ULK2, UVRAG, WDR45, and WIPI1 mRNA expression were assessed by $\mathrm{qPCR}$ and were normalized to HPRT1 mRNA levels, used as internal control. Data display the foldchanges relative to control cells ( $n=3-4-5-6$, based on the variation of each gene expression) and are expressed as the mean value \pm SEM. C Summary of those genes that were significantly upregulated in both cell lines upon starvation (STV), upon AZD8055 treatment (AZD) or upon both conditions (both). D List of genes for the evaluation of lysosomal biogenesis. Statistical analysis was performed using one-way ANOVA with Dunnett's multiple comparisons test. $\left({ }^{*} p<0.05 ;{ }^{* *} p<0.01 ;{ }^{* *} p<0.001 ;{ }^{* * *} p<0.0001\right)$.

experimentally examined $[15,18,19,28-30]$. Emerging evidence suggest that modulation of these targets is mostly coordinated by the nuclear translocation of members of the microphthalmia/ transcription factor $\mathrm{E}$ (MiT/TFE) family that includes TFEB, TFE3, MITF, and TFEC [38].

Of note, we have built this comprehensive list of autophagy genes to be particularly useful during an effective RNAsequencing analysis. Thus, to show its applicability, we performed a gene set enrichment analysis (GSEA) $[39,40]$ on two previously published RNA-seq datasets, by using the "autophagy core" list: in a first case, RNA-seq was achieved on the human HAP1 cell line, deprived of amino acids and serum for $6 \mathrm{~h}$ in EBSS, in which autophagy activation was clearly demonstrated by measuring the autophagic flux [31], and hence represents an excellent positive control. In a second case, the RNA-seq was performed on primary fibroblasts derived from Down Syndrome patients, in which we identified a mitophagy and autophagy deficit associated with mTORC1 hyperactivation [27]; by contrast with the first case, this can therefore be considered as a negative control. The "autophagy core" list was cleaned from the "Negative Regulator of Autophagy" 
A

Autophagy core list upon $6 \mathrm{~h}$ of Starvation

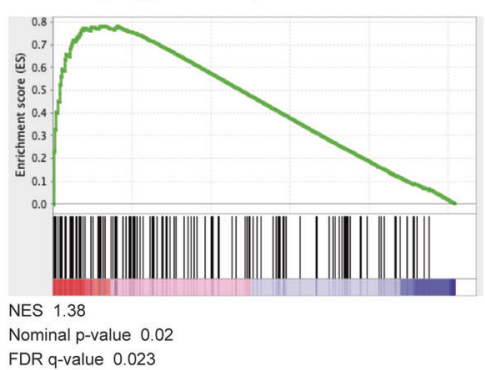

B

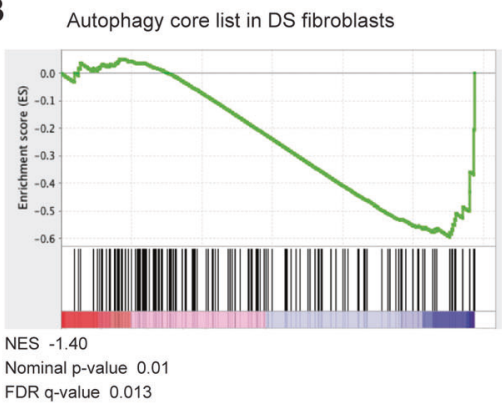

Fig. 4 Experimental validation of the "autophagy core" list. GSEA analysis obtained by using the "autophagy core" list, cleaned of "Negative Regulator of Autophagy" and "Its mutation leads to Autophagy defects" sub-categories, in starved HAP1 cells (A) or in DS fibroblasts compared to control fibroblast (B).

and the "Its mutation leads to Autophagy defects" sub-categories, to better evaluate genes positively regulated upon autophagy induction. Intriguingly, the "autophagy core" list was significantly enriched in starved cells (Normalized Enrichment Score, NES, 1.38, Nominal p-value 0.02, FDR q-value 0.023, Fig. 4A and Supplementary $3 \mathrm{~A})$. By contrast, the same list was negatively enriched in DS fibroblasts compared to $2 \mathrm{~N}$ control cells (NES -1.40, Nominal p-value 0.01, FDR q-value 0.013, Fig. 4B and Supplementary 3B), thus demonstrating that our list can be easily applied for evaluation of the autophagy state throughout GSEA analysis. Moreover, by analyzing the expression profile of genes that compose the "induction list", 15 out of 20 were upregulated in starved cells, including ATG14, NBR1, ULK1, and WDR45 (Supplementary Table 2) that we observed to be increased both upon starvation and mTORC inhibition, together with ATG16L1, ATG3, PINK1，BCL2，GABARAPL1，WIPI1，SQSTM1， OPTN, MAP1LC3B, UVRAG, GABARAP. Only DS fibroblasts showed the upregulation of MAP1LC3B, but, on the contrary, 17 targets did not change, and ATG7 and GABARAPL1 were highly downregulated, as previously indicated [27]. Thus, in the presence of mTORC1 activation and consequent autophagy inhibition, these targets are mostly unchanged or downregulated. Next, we evaluated the genes included in the "lysosomal biogenesis" list: in this case, starvation leads to the positive regulation of 13 out of 26 targets (including LAMP1, MCOLN1, and ATP6VOD1), while 3 genes were downregulated, suggesting that -most likely, $6 \mathrm{~h}$ starvation may not be sufficient to properly boost the expression of lysosomal genes (Supplementary Table 2), at least in these cells. We earlier reported that DS fibroblasts exhibit a perturbation of lysosomal activities driven by APP- $\beta C T F$-dependent compromised luminal acidification [41]; interestingly, in these cells, among the "lysosomal biogenesis" genes, most of them (18) were not changed, while 7 were upregulated and 1 downregulated, CLCN7, which was found to be upregulated under starvation (Supplementary Table 2). Of note, in some instances, such as different cell types or stimuli, we could not rule out the occurrence of an earlier autophagy activation (less than $4 \mathrm{~h}$ from a given stimulus). Thus, a timecourse experiment was performed to evaluate ATG14, NBR1, ULK1, and WDR45 levels upon starvation or mTOR inhibition in the 30 min- $2 \mathrm{~h}$ time window. This analysis revealed that these genes showed increased expression at 2 hours only in SH-SY5Y cells, and only upon AZD8055 treatment (Supplementary Figure 4), and thus that short time windows may turn out to be generally insufficient for a robust regulation of gene expression at a transcriptional level.

\section{DISCUSSION}

By our study, we generated a very relevant toolbox for the study of autophagy in a number of interesting conditions, by applying RNA-seq. Nowadays, RNA-seq analysis is increasingly accessible and is largely used for studying biological samples; through the pathway enrichment analysis, researchers can indeed more easily discover or interpret pathways in relation to disease mechanisms or to specific experimental conditions, such as gene KO. Over recent years, the relevance of autophagy and selective autophagy in the context of human pathophysiology has considerably grown. In fact, age- or genetic-related autophagy dysfunction is associated with an extensive number of disorders, such as cancer, diabetes and neurodegenerative diseases [3, 9, 10, 38]. For example, the role of autophagy in cancer cell fate determination is particularly dependent on the cell type or tissue, on the stage of tumor's development and on the environment (recently reviewed in [42]). Indeed, in this context, it becomes critical to correctly evaluate the potential alteration of a specific autophagy status or step, for understanding whether at all and how autophagy is involved, and then for developing therapies capable of manipulating single mechanisms of autophagy or autophagy regulation. Our comprehensive autophagy transcriptional toolbox is thus highly versatile: it can be easily applied for enrichment analysis by using the entire list or, in alternative, shorter lists can be derived focusing the investigation on selective complexes or functions of the process; Also, one could get useful insights about a putative autophagy status, as demonstrated by re-analyzing published data-sets running the "autophagy core" list. Although the main list is a valuable resource to analyze autophagy in depth, the validated activation-list, made by 20 targets, can also be a very efficient tool. Interestingly, by Real Time PCR upon mTOR inhibition or upon starvation, we identified 6 genes that are fundamental for the proper initiation of the autophagy cascade: ATG14 is a component of the class III PI3K complex 1(PI3KC3-C1), formed by the catalytic subunit vacuolar protein sorting 34 (VPS34), Beclin 1 and general vesicular transport factor $\mathrm{p} 115$. This complex activates the phosphatidylinositol-3-phosphate (PI3P) production on the origin site of omegasome [3]. ATG7 is strictly required for autophagosome formation; it is an E1-like activating enzyme present in ubiquitin-like conjugation systems that promotes both cleavage and lipidation of LC3 and GABARAP [43]. NBR1 is an autophagy receptor binding ubiquitylated cargo and recent studies demonstrated that it is also required for selective degradation of peroxisomes (pexophagy) and of ubiquitylated aggregates (aggrephagy) [44]. Intriguingly, NBR1 expression is highly responsive to autophagy activation, more than 3-fold increase compared to control, implying that its promoter is heavily regulated upon nutrient deprivation or mTOR inhibition. ULK1 (Unc-51-like kinase 1) is a Serine/threonine kinase that, together with RB1-inducible coiled-coil protein 1 (FIP200), ATG13, and ATG101 forms the ULK1 complex. Accordingly, ULK1 is considered as the master regulator of autophagy induction, triggering a cascade of signals, through direct phosphorylation of ATG proteins, that culminate in autophagosome formation [3, 43]. Its activation is accurately regulated by mTORC1 and AMPK activity [3]. Furthermore, recent evidence indicates that the 
ULK2 isoform is a functionally redundant autophagic protein kinase and is ubiquitously expressed such as ULK1 [45]. As ULK1, ULK2 can promote autophagy response following starvation [45], indicating that also this isoform can be informative for the analysis. Obviously, the fact that ULK proteins are kinases, highlights a scenario, in which post-translational modification analysis should integrate in a number of instances, the approach proposed here. Nevertheless, also WDR45 (WD repeat domain 45), that has been found to be important for autophagosome formation, exhibited an excellent positive regulation in the presence of autophagy induction, even though its specific function remains yet unclear [46]. However, other genes, fundamental for the formation and maturation of the autophagosome, i.e., ATG3, ATG9A, GABARAP, MAP1LC3B, UVRAG, and WIPI1 are significantly up-regulated only upon starvation, while mTOR inhibition exerts significant changes in ATG10 and OPTN expression but not on previous gene groups, or at least not in both cell lines. Of note, RNA-seq analysis on starved human HAP1 cells revealed that, besides ATG14, NBR1, ULK1, and WDR45, also ATG16L1, ATG3, PINK1, BCL2, GABARAPL1, WIPI1, SQSTM1, OPTN, MAP1LC3B, UVRAG, GABARAP were significantly upregulated, while upon mTOR hyperactivation, almost all these targets did not show relevant modifications, with the exception of ATG7 and GABARAPL1 (downregulated) or MAP1LC3B (upregulated). Thus, irrespective of the cell type, we may conclude that ATG14, NBR1, ULK1, and WDR45 are high reliable markers for evaluation of autophagy activation, whereas other targets of the induction list may or may not show an adequate modulation in response to autophagy stimulation; this indicates, as it might be expected, the existence of cell-specific regulatory pathways, which in any case do not compromise the reliability of our list. In fact, in DS cells, in which autophagy and mitophagy induction are impaired [27], most of these targets were unchanged or downregulated, with the only exception of MAP1LC3B. Furthermore, during fasting or mTOR inhibition, activation of MiT/TFE transcription factors occurs, with this fostering lysosomal gene expression [47]; again, the appropriate tuning of lysosomal gene expression is strictly related to the duration of the stimuli and to the cell type. In HAP1 cells, after $6 \mathrm{~h}$ of starvation, $50 \%$ of the genes included in our literaturebased lysosome biogenesis list were upregulated, indicating that -most likely, the molecular regulation underlying lysosomal biogenesis requires more prolonged starvation in this cell line. Intriguingly, despite a reduction in lysosomal activity [41], DS cells showed the upregulation of 7 lysosomal targets; this might be explained by the existence of a possible compensatory mechanism or by the effect of other overexpressed genes localized on chromosome 21. Collectively, our research efforts were directed toward developing an inclusive and accurate autophagy transcription gene list functional to autophagy assessment and further identification of autophagy dysfunctions in disease. Thus, discerning the autophagy roles may direct efforts to design therapy strategies based on the adequate modulation of autophagy. Importantly, as we proved by two different approached, additional step-specific sub-lists could be generated by experimental easy approaches or even by digging into solid literature databases, which should include established experimental data.

\section{MATERIALS AND METHODS \\ Generation of the list}

The comprehensive autophagic gene list was derived from a broad literature analysis. The list is composed by 646 genes, divided in 6 main categories, as reported in the text. In each category, we created subgroups based on their belonging to the same complex or to a specific regulatory pathway. To this end, we reported at least one reference that has been used to identify the function of each single gene. The references are indicated in the column A of the excel file; the column B of the file states the Biological Function, while the Column C, D, and E display the official gene symbol, the common name of the related protein (alias), and the description, respectively. Since some genes are present in more than one group, due to their multiple functions along the autophagy process, these genes are listed more than once and are marked with the red color. The restricted "induction list" for the prediction of autophagy activation state, made by 20 targets, was obtained combining data from previous publications [18-27], in which have been documented the increase of the mRNA expression following autophagy induction. Similarly, the list for the evaluation of "lysosomal biogenesis" was created from literature by combining data that underwent preceding experimental validation $[15,18,19,28-30]$.

\section{Cell culture and treatments}

The human embryonic kidney HEK293 cell line were cultured in Dulbecco's modified Eagle's medium (DMEM, Euroclone), while human neuroblastoma SHSY-5Y cell line in DMEM-F12 $(1: 1$, Gibco $)$ at $37^{\circ} \mathrm{C}$ under $5 \% \mathrm{CO}_{2}$; both mediums were supplemented with $10 \%$ heat inactivated fetal bovine serum (FBS, Gibco), 2mM L-glutamine, $100 \mathrm{U} / \mathrm{ml}$ penicillin and $100 \mathrm{mg} / \mathrm{ml}$ streptomycin (Gibco). The induction of autophagy by nutrients starvation was obtained by incubating HEK293 cells with Earle's balanced salt solution (EBSS; Sigma-Aldrich) for $4 \mathrm{~h}$ and SHSY-5Y cells for $8 \mathrm{~h}$. In the other case, autophagy stimulation was obtained by treating cells with $100 \mathrm{nM}$ AZD8055 (AZD), an ATP-competitive inhibitor directly targeting the mTOR catalytic site (Selleckchem) [37], for $8 \mathrm{~h}$. Both cells lines were also starved or treated with AZD8055 for $30 \mathrm{~min}, 1 \mathrm{~h}, 1.5 \mathrm{~h}$, and $2 \mathrm{~h}$, as indicated in figure legend.

\section{Preparation of CDNA and Real-Time (qPCR)}

RNA was extracted from cells using ReliaPrep ${ }^{T M}$ RNA Cell Miniprep System (Promega) according to the manufacturer's specifications, including DNase treatment step. RNA quantity was determined with a Nanodrop 1000 spectrophotometer (Wilmington, DE). CDNA was prepared from total RNA using GoScript ${ }^{\mathrm{TM}}$ Reverse Transcription Mix according to manufacturer's instructions and using Random Primers (Promega). Following reverse transcription, qPCR was then performed using SYBR Green Mix (Thermo Fisher Scientific) with the STEPONE Real-Time PCR System (Applied Biosystems $\mathrm{s}^{\mathrm{TM}}$ ). The oligos primers are listed in Supplementary Table 3.

The target genes were normalized against HPRT1 that was used as a housekeeping gene. Results were calculated using the $\Delta \Delta \mathrm{Ct}$ method. We then normalized the mRNA levels to the control condition. Reported values are the means of at least three independent biological replicates with technical triplicates that were averaged for each experiment. Error bars represent the SEM of the mean.

\section{Gene set and ontology enrichment analysis}

Gene set enrichment analyses were performed according to standard procedures $[39,40]$ on two published RNA-seq datasets [31, 48]; GSEA was run by using the "autophagy core" list from Supplementary Table 1, cleaned of "Negative Regulator of Autophagy" and "Its mutation leads to Autophagy defects" sub-categories. The Gene Ontology Enrichment Analysis (GOEA) $[49,50]$ was performed by using the DAVID online tool (DAVID Bioinformatics Resources 6.8) [51] restricting the output to Biological Process terms (BP_FAT), Cellular Compartment terms (CC_FAT), and Molecular Function terms (MF_FAT). GOEA results are reported in Supplementary Table 4. In Supplementary Figure 3, we plotted the top 10 BP, CC and MF significantly enriched terms. The threshold for statistical significance of GOEA was FDR $<0,1$ and the Enrichment Score $\geq 1.5$. Heatmaps were generated using GraphPad Prism program (version 7), showing the log2fold change of each gene.

\section{Statistical analysis}

Statistical significance was measured using one-way ANOVA with Dunnett's multiple comparisons test calculated with GraphPad Prism program (version 7), as reported in the figure legend.

\section{REFERENCES}

1. Yu L, Chen $\mathrm{Y}$, Tooze SA. Autophagy pathway: cellular and molecular mechanisms. Autophagy. 2018;14:207-15.

2. Galluzzi L, Baehrecke EH, Ballabio A, Boya P, Bravo-San Pedro JM, Cecconi F, et al. Molecular definitions of autophagy and related processes. EMBO J. 2017;36:1811-36. 
3. Death C, Dikic I. Mechanism and medical implications of mammalian autophagy. Nat Rev Mol Cell Biol. 2018;19:349-64.

4. Liu GY, Sabatini DM mTOR at the nexus of nutrition, growth, ageing and disease. Nat Rev Mol Cell Biol. 2020; 8. https://doi.org/10.1038/s41580-019-0199-y.

5. Herzig S, Shaw RJ. AMPK: Guardian of metabolism and mitochondrial homeostasis. Nat Rev Mol Cell Biol. 2018;19:121-35.

6. Pickles S, Vigié P, Youle RJ. Mitophagy and quality control mechanisms in mitochondrial maintenance. Curr Biol. 2018;28:R170-R185.

7. Holdgaard SG, Cianfanelli V, Pupo E, Lambrughi M, Lubas M, Nielsen JC, et al. Selective autophagy maintains centrosome integrity and accurate mitosis by turnover of centriolar satellites. Nat Commun. 2019;10:1-19.

8. Klionsky DJ, Abdelmohsen K, Abe A, Abedin MJ, Abeliovich H, Acevedo Arozena $A$, et al. Guidelines for the use and interpretation of assays for monitoring autophagy (3rd edition). Autophagy. 2016;12:1-222.

9. Hansen M, Rubinsztein DC, Walker DW. Autophagy as a promoter of longevity: insights from model organisms. Nat Rev Mol Cell Biol. 2018;19:579-93.

10. Nixon RA. The role of autophagy in neurodegenerative disease. Nat Med. 2013;19:983-97.

11. White E. The role for autophagy in cancer. J. Clin. Investig. 2015; 125: 42-46.

12. Reimand J, Isserlin R, Voisin V, Kucera M, Tannus-lopes C, Rostamianfar A, et al. Pathway enrichment analysis and visualization of omics data using g: Pro fi ler, GSEA, Cytoscape and EnrichmentMap. Nat Protoc. 2019;14:482-517.

13. Polishchuk EV, Merolla A, Lichtmannegger J, Romano A, Indrieri A, llyechova EY, et al. Activation of autophagy, observed in liver tissues from patients with wilson disease and from ATP7B-deficient animals, protects hepatocytes from copperinduced apoptosis. Gastroenterology. 2019;156:1173-89.

14. Pecoraro A, Carotenuto P, Franco B, De Cegli R, Russo G, Russo A Role of uL3 in the crosstalk between nucleolar stress and autophagy in colon cancer cells. Int $J$ Mol Sci. 2020; 21. https://doi.org/10.3390/ijms21062143.

15. Fruscio Di G, Schulz A, Cegli De R, Savarese M, Mutarelli M, Parenti G, et al. Lysoplex: An efficient toolkit to detect DNA sequence variations in the autophagy-lysosomal pathway. Autophagy. 2015;11:928-38.

16. Deng W, Ma L, Zhang Y, Zhou J, Wang Y, Liu Z THANATOS: an integrative data resource of proteins and post-translational modifications in the regulation of autophagy. Autophagy 2018; 14. https://doi.org/10.1080/15548627.2017.1402990.

17. Wang NN, Dong J, Zhang L, Ouyang D, Cheng Y, Chen AF, et al. HAMdb: a database of human autophagy modulators with specific pathway and disease information. J Cheminform. 2018;10:1-8.

18. Settembre C, Polito VA, Arencibia MG, Vetrini F, Erdin S, Erdin SU, et al. TFEB links autophagy to lysosomal biogenesis. Science. 2011;332:1429-33.

19. Martina JA, Diab HI, Lishu L, Jeong-A L, Patange S, Raben N, et al. The nutrientresponsive transcription factor TFE3 promotes autophagy, lysosomal biogenesis, and clearance of cellular debris. Sci Signal. 2014;7:ra9.

20. Chandra V, Bhagyaraj E, Parkesh R, Gupta P Transcription factors and cognate signalling cascades in the regulation of autophagy. Biol Rev 2015. https://doi.org/ 10.1111/brv.12177.

21. B'Chir W, Maurin AC, Carraro V, Averous J, Jousse C, Muranishi Y, et al. The elF2a/ ATF4 pathway is essential for stress-induced autophagy gene expression. Nucleic Acids Res. 2013;41:7683-99.

22. Klinkenberg M, Gispert S, Dominguez-Bautista JA, Braun I, Auburger G, Jendrach $M$. Restriction of trophic factors and nutrients induces PARKIN expression. Neurogenetics. 2012;13:9-21.

23. Wise JP, Cannon J Alterations in Optineurin Expression and Localization in Preclinical Parkinson's Disease Models. Toxicol Sci. 2016. https://doi.org/10.1093/ toxsci/kfw133.

24. Kenzelmann Broz D, Mello SS, Bieging KT, Jiang D, Dusek RL, Brady CA, et al. Global genomic profiling reveals an extensive p53-regulated autophagy program contributing to key p53 responses. Genes Dev. 2013;27:1016-31.

25. Devenish RJ, Prescott M. Autophagy: starvation relieves transcriptional repression of ATG genes. Curr Biol. 2015;25:R238-R240.

26. Tsuyuki S, Takabayashi M, Kawazu M, Kudo K, Watanabe A, Nagata $Y$, et al. Detection of WIPI1 mRNA as an indicator of autophagosome formation. Autophagy. 2014;10:497-513.

27. Bordi $M$, Darji $S$, Sato $Y$, Mellén $M$, Berg $M J$, Kumar $A$, et al. mTOR hyperactivation in Down Syndrome underlies deficits in autophagy induction, autophagosome formation, and mitophagy. Cell Death Dis. 2019;10:563.

28. Sardiello M, Palmieri M, di Ronza A, Medina DL, Valenza M, Gennarino VA, et al. A gene network regulating lysosomal biogenesis and function. Science. 2009;325:473-7.

29. Palmieri M, Impey S, Kang H, di Ronza A, Pelz C, Sardiello M, et al. Characterization of the CLEAR network reveals an integrated control of cellular clearance pathways. Hum Mol Genet. 2011;20:3852-66.

30. Song W, Wang F, Savini M, Ake A, Di Ronza A, Sardiello M, et al. TFEB regulates lysosomal proteostasis. Hum Mol Genet. 2013;22:1994-2009.
31. Peeters JGC, Picavet LW, Coenen SGJM, Mauthe M, Vervoort SJ, Mocholi E, et al. Transcriptional and epigenetic profiling of nutrient-deprived cells to identify novel regulators of autophagy. Autophagy. 2019;15:98-112.

32. Jiang $P$, Nishimura $T$, Sakamaki $Y$, Itakura $E$, Hatta $T$, Natsume $T$, et al. The HOPS complex mediates autophagosome-lysosome fusion through interaction with syntaxin 17. Mol Biol Cell. 2014;25:1327-37.

33. Ao X, Zou L, Wu Y. Regulation of autophagy by the Rab GTPase network. Cell Death Differ. 2014;21:348-58.

34. Harper JW, Paulo JA, Shen K, Heo J-M, Sabatini DM, Ordureau A, et al. RAB7A phosphorylation by TBK1 promotes mitophagy via the PINK-PARKIN pathway. Sci Adv. 2018:4:eaav0443.

35. Ni H, Williams JA, Ding W. Redox Biology Mitochondrial dynamics and mitochondrial quality control. Redox Biol. 2015;4:6-13.

36. Nezich CL, Wang C, Fogel Al, Youle RJ. MiT / TFE transcription factors are activated during mitophagy downstream of Parkin and Atg5. J Cell Biol. 2015;210:435-50.

37. Chresta CM, Davies BR, Hickson I, Harding T, Cosulich S, Critchlow SE, et al. AZD8055 is a potent, selective, and orally bioavailable ATP-competitive mammalian target of rapamycin kinase inhibitor with in vitro and in vivo antitumor activity. Cancer Res. 2010;70:288-98.

38. Di Malta $C$, Cinque L, Settembre C. Transcriptional regulation of autophagy: mechanisms and diseases. Front Cell Dev Biol. 2019;7:1-10.

39. Subramanian A, Tamayo P, Mootha VK, Mukherjee S, Ebert BL, Gillette MA, et al. Gene set enrichment analysis: a knowledge-based approach for interpreting genome-wide expression profiles Aravind. Proc Natl Acad Sci USA. 2005;102:15545-50.

40. Liberzon A, Birger C, Thorvaldsdóttir H, Ghandi M, Mesirov JP, Tamayo P. The molecular signatures database hallmark gene set collection. Cell Syst. 2015;1:417-25.

41. Jiang $Y$, Sato $Y$, Im E, Berg $M$, Bordi $M$, Mohan PS et al. Lysosomal dysfunction in Down syndrome is APP-dependent and mediated by APP- $\beta C T F$ (C99). J Neurosci. 2019.

42. Levy JMM, Thorburn A. Autophagy in cancer: moving from understanding mechanism to improving therapy responses in patients. Cell Death Differ. 2020;27:843-57.

43. Feng Y, He D, Yao Z, Klionsky DJ. The machinery of macroautophagy. Cell Res. 2014;24:24-41.

44. Mancias JD, Kimmelman AC. Mechanisms of selective autophagy in normal physiology and cancer. J Mol Biol. 2016:428:1659-80.

45. Lee $E$, Tournier $C$. The requirement of uncoordinated 51-like kinase 1 (ULK1) and ULK2 in the regulation of autophagy. Autophagy. 2011;7:689-95.

46. Saitsu H, Nishimura T, Muramatsu K, Kodera H, Kumada S, Sugai K. et al. De novo mutations in the autophagy gene WDR45 cause static encephalopathy of childhood with neurodegeneration in adulthood. Nat Publ Gr. 2013;45:445-9.

47. Sardiello M. Transcription factor EB: from master coordinator of lysosomal pathways to candidate therapeutic target in degenerative storage diseases. Ann N. Y Acad Sci. 2016;1371:3-14.

48. Bordi M, Berg MJ, Mohan PS, Peterhoff CM, Alldred MJ, Che S, et al. Autophagy flux in CA1 neurons of Alzheimer hippocampus: Increased induction overburdens failing lysosomes to propel neuritic dystrophy. Autophagy. 2016;12:1-17.

49. Huang DW, Lempicki RA, Sherman BT. Systematic and integrative analysis of large gene lists using DAVID bioinformatics resources. Nat Protoc. 2009;4:44-57.

50. Jiao X, Sherman BT, Huang DW, Stephens R, Baseler MW, Lane HC, et al. DAVIDWS: a stateful web service to facilitate gene/protein list analysis. Bioinformatics. 2012;28:1805-6

51. Dennis G, Sherman BT, Hosack DA, Yang J, Gao W, Lane C, et al. DAVID: database for annotation, visualization, and integrated discovery. Genome Biol. 2003;4:60.

\section{ACKNOWLEDGEMENTS}

We thank G. Napolitano for critical reading of the manuscript and for his valuable suggestions. Funding statement: M.B. was supported by a "Fondazione Umberto Veronesi" fellowship. A.B. is supported by the Telethon Foundation, the European Research Council (ERC) advanced grant ERC-LYSOSOMICS, NIH ROI-NS078072, and AIRC IG 2015 ID I7639.

\section{AUTHOR CONTRIBUTIONS}

M.B. and F.C. conceived the study and designed experiments. R.D.C. and B.T. performed experiments. R.A.N. and A.B. gave experimental support and conceptual advice. M.B. and F.C. wrote the original draft.

\section{ETHICS STATEMENT}

No human participants, human tissue, or animal model is involved in this study. 


\section{COMPETING INTERESTS}

Andrea Ballabio is a Co-Founder of CASMA Therapeutics.

\section{ADDITIONAL INFORMATION}

Supplementary information The online version contains supplementary material available at https://doi.org/10.1038/s41419-021-04121-9.

Correspondence and requests for materials should be addressed to Francesco Cecconi.

Reprints and permission information is available at http://www.nature.com/ reprints

Publisher's note Springer Nature remains neutral with regard to jurisdictional claims in published maps and institutional affiliations.
Open Access This article is licensed under a Creative Commons

Attribution 4.0 International License, which permits use, sharing,


appropriate credit to the original author(s) and the source, provide a link to the Creative Commons license, and indicate if changes were made. The images or other third party material in this article are included in the article's Creative Commons license, unless indicated otherwise in a credit line to the material. If material is not included in the article's Creative Commons license and your intended use is not permitted by statutory regulation or exceeds the permitted use, you will need to obtain permission directly from the copyright holder. To view a copy of this license, visit http://creativecommons. org/licenses/by/4.0/.

(c) The Author(s) 2021 